\title{
Integrated analysis of coding genes and non-coding RNAs during hair follicle cycle of cashmere goat (Capra hircus)
}

\author{
Shanhe Wang ${ }^{1}$, Wei Ge1', Zhixin Luo ${ }^{1}$, Yang Guo ${ }^{1}$, Beilei Jiao ${ }^{1}$, Lei $\mathrm{Qu}^{2}$, Zhiying Zhang ${ }^{1}$ and Xin Wang ${ }^{1 *}$
}

\begin{abstract}
Background: Cashmere growth is a seasonal and cyclic phenomenon under the control of photoperiod and multiple stimulatory and inhibitory signals. Beyond relevant coding genes, microRNA (miRNA) and long non coding RNA (IncRNA) play an indispensable role in hair follicle (HF) development and skin homeostasis. Furthermore, the influence of IncRNA upon miRNA function is also rapidly emerging. However, little is known about miRNAs, IncRNAs and their functions as well as their interactions on cashmere development and cycling.

Result: Here, based on IncRNA and miRNA high-throughput sequencing and bioinformatics analysis, we have identified 1108 IncRNAs and 541 miRNAs in cashmere goat skin during anagen and telogen. Compared with telogen, 1388 coding genes, 41 IncRNAs and 15 miRNAs were upregulated, while 1104 coding genes, 157 IncRNAs and 8 miRNAs were downregulated in anagen (adjusted $P$-value $\leq 0.05$ and relative fold-change $\geq 2$ ). Subsequently, we investigated the impact of IncRNAs on their target genes in cis and trans, indicating that these IncRNAs are functionally conserved during HF development and cycling. Furthermore, miRNA-mRNA and miRNA-IncRNA interaction were identified through the bioinformatics algorithm miRanda, then the ceRNA networks, miR-221-5p-Inc_000679-WNT3, miR-34a-Inc_000181-GATA3 and miR-214-3p-Inc_000344-SMAD3, were constructed under defined rules, to illustrate their roles in cashmere goat HF biology.

Conclusion: The present study provides a resource for IncRNA, miRNA and mRNA studies in cashmere cycling and development. We also demonstrate potential ceRNA regulatory networks in cashmere goat HF cycling for the first time. It expands our knowledge about IncRNA and miRNA biology as well as contributes to the annotation of the goat genome.
\end{abstract}

Keywords: Cashmere goat, Anagen, Telogen, Hair follicle, IncRNA, miRNA

\section{Background}

Capra hircus, an economically important livestock species, plays an indispensable role in the world animal fiber industry [1]. Shanbei white cashmere goat, a double-coated (wool and cashmere) species famous for its high and luxurious fiber production traits, exhibits seasonal rhythms with a well-defined duration of fiber growth and have been an excellent cashmere goat breed in China.

\footnotetext{
*Correspondence: wxwza@126.com

${ }^{1}$ College of Animal Science \& Technology, Northwest A\&F University,

Yangling, Shaanxi 712100, China

Full list of author information is available at the end of the article
}

Cashmere growth is a seasonal phenomenon under the control of photoperiod and the animals' endocrine systems [2-4]. The cashmere of the Shanbei white cashmere goat undergoes cyclic transformation from the resting phase (telogen, May to July, summer) to the growth phase (anagen, July to February). The latter phase is characterized by rapid proliferation of follicular keratinocytes and elongation and thickening of the hair shaft. The regression phase (catagen, March to May, spring) leads to the degeneration of the HF. The basis for HF cycle rests in the unique follicular epithelial and mesenchymal components and their interactions $[5,6]$. Recently, some molecular signals such as fibroblast 
growth factor, transforming growth factor- $\beta$, WNT signaling pathway, sonic hedgehog, neurotrophins, and homeobox [7-11], and their interactions have been defined [12-14]. These gene families were also found in other regenerating systems $[15,16]$.

The recent explosion in knowledge demonstrating the importance of miRNA and IncRNA in the regulation of multiple major biological processes impacting development, differentiation, and metabolism have brought these heretofore neglected molecular players to the forefront [17-19]. Specifically, for the biology of HF, the miRNA-processing enzyme Dicer was essential for the morphogenesis and maintenance of HF [20]. Meanwhile, another miRNA-processing enzyme Drosha was required for HF regression, hair shaft differentiation, long-term maintenance of HF stem cells, and epidermal homeostasis [21]. Since then more miRNAs have been identified and characterized in HF biology, such as miR-214, miR21 and miR-24 [22-24]. Researchers have identified several functional lncRNAs associated with skin biology, such as ANCR, TINCR, U1 RNA, PRINS, BANCR, and SPRY4-IT1 [25]. Furthermore, IncRNA in dermal papilla cells contributes to regulating the genes involved in hair follicle development and postnatal hair cycling [26]. Even more important, the influence of lncRNAs on microRNA function is also rapidly emerging [27-29], the microRNA-IncRNA regulatory paradigms modulate gene expression patterns that drive major cellular processes (such as cell differentiation, proliferation, and cell death) which are central to mammalian physiologic and pathologic processes [30, 31].

However, previous findings mainly focused on human or mouse, little was known about miRNAs, lncRNAs and their functions on cashmere development and cycling $[19,32]$. In this current study, the coding genes, lncRNA as well as miRNA profile of cashmere goat skin in anagen and telogen were detected using deepsequencing. Subsequently, the function of key genes, miRNAs, lncRNAs and their potential interactions on cashmere development and cycling were analyzed using bioinformatics. This study will expand our knowledge about lncRNA and miRNA in HF biology as well as contribute to the annotation of the goat genome.

\section{Methods}

\section{Animals and samples}

Shanbei cashmere goats with the fine fiber production trait were used in this study. All the goats were obtained from Shanbei cashmere goats engineering technology research center of Shaanxi province, China. The experimental animals were fed according to the local cashmere goat standard of Shaanxi (DB61/T583-2013, http://www.sxny. gov.cn/). Six female adults (1 year old, coefficient of relationship $<0.125)$ were selected. After intravenous injection of lidocaine hydrochloride, skin samples approximately $2 \mathrm{~cm}^{2}$ and $3 \mathrm{~mm}$ deep were harvested from the body side of adult goats at distinct hair cycle stages (anagen and telogen), frozen in sample protector for RNA (Takara, China) and stored at $-80{ }^{\circ} \mathrm{C}$ for future analysis. The same animals were collected at an adjacent site at both anagen and telogen.

All the experimental procedures with goats used in the present study had been given prior approval by the Experimental Animal Manage Committee of Northwest A\&F University (2011-31101684).

\section{RNA isolation, library preparation, and sequencing}

Total RNA was extracted from the collected skin tissues using Trizol reagent (Invitrogen, USA) following the manufacturer's instructions, after grinding them in liquid nitrogen. The RNA concentration and quality was determined using the Agilent 2100 Bioanalyzer. The extracted total RNA was stored at $-80{ }^{\circ} \mathrm{C}$ for later use.

For lncRNA sequencing, a total amount of $3 \mu \mathrm{g}$ RNA per sample was used as input material for the RNA library preparations. Firstly, ribosomal RNA was removed using the Epicentre Ribo-zero ${ }^{\text {Tu }}$ rRNA Removal Kit (Epicentre, USA), and the rRNA was cleaned up by ethanol precipitation. Subsequently, in total six libraries from anagen $(n=3)$ and telogen $(n=3)$ were generated from the rRNA-depleted RNA using the NEBNext ${ }^{\bullet}$ Ultra $^{\text {ax }}$ Directional RNA Library Prep Kit for Illumina (NEB, USA) following the manufacturer's recommendations. Strand-specific sequencing was performed on the Illumina Hiseq 4000, PE 150 system for these libraries (paired-end 100-bp reads).

For miRNA sequencing, the six animals at each stage were randomly divided into two groups, and the RNA from three single goats per group was pooled. Four RNA pool libraries from telogen $(n=2)$ and anagen $(n=2)$ were constructed. Small RNA fragments of 18-30 nt in length were isolated and purified from total RNA using $15 \%$ denaturing polyacrylamide gel electrophoresis (PAGE) using a gel extraction kit (Sangon Biotech, China). Subsequently, $3^{\prime}$ and 5' RNA adaptors were ligated to the RNA pool using NEBNext ${ }^{\circ}$ Multiplex Small RNA Library Prep Set for Illumina ${ }^{\circ}$ (NEB, USA.) following the manufacturer's recommendations, and index codes were added to attribute sequences to each sample. Then, first-strand cDNA was synthesized using M-MuLV Reverse Transcriptase (RNase H-) (NEB, USA.). PCR amplification was performed using LongAmp Taq $2 \times$ Master Mix (NEB, USA.), SR Primer for illumina and index (X) primer for 16 cycles. PCR products were purified on a $10 \%$ polyacrylamide gel (100 V, $80 \mathrm{~min})$. DNA fragments corresponding to $140 \sim 160$ bp (the length of small noncoding RNA plus the $3^{\prime}$ and $5^{\prime}$ adaptors) were recovered and rehydrated in 
$8 \mu \mathrm{L}$ elution buffer (OMEGA bio tek, USA.). Then, library quality was assessed on the Agilent Bioanalyzer 2100 system using DNA High Sensitivity Chips. The four library preparations were sequenced on an Illumina Hiseq 2500 platform and $50 \mathrm{bp}$ single-end reads were generated.

\section{Quality control}

Raw data were first processed using in-house Perl scripts. In this step, clean data were obtained by trimming reads containing adapter, reads containing over $10 \%$ of ploy- $\mathrm{N}$, and low-quality reads $(>50 \%$ of bases whose Phred scores were $<5)$ from the raw data. The Phred score [33] (Q20, Q30) and GC content of the clean data were calculated. All subsequent analysis was based on the high-quality data.

\section{Transcriptome assembly}

The high quality reads were mapped independently to the goat genome v2.0 (ftp://ftp.ncbi.nlm.nih.gov/genomes/all/GCA/000/317/765/GCA_000317765.2_CHIR_ 2.0) using Bowtie v2.0.6 [34] and the spliced read aligner TOPHAT v2.0.9 (main parameter: library-type $<$ fr- firststrand $>$ ) [35]. The mapped reads of each sample were assembled using Cufflinks (v2.1.1) in a reference-based approach [36]. Cufflinks was run with'min-frags-pertransfrag $=0$ ' and '-library-type', other parameters were set as default. We then adopted five steps to identify goat lncRNAs from the assembled transcripts: (1) transcripts with length $<200 \mathrm{bp}$ were removed; (2) transcripts with exon number $<2$ were removed; (3) transcripts were compared with mRNA, rRNA, tRNA, snRNA, snoRNA and pre-miRNA (https://www.ncbi.nlm.nih.gov/) using Cuffcompare v2.1.1 to remove the same or similar transcripts [36]. (4) transcripts with FPKM $<0.5$ were removed; (5) transcripts that did not pass the protein-coding-score test were removed using the Coding Potential Calculator (CPC) [37], PFAM database [38] and Coding-Non-Coding Index (CNCI) software [39]. CNCI was used with default parameters. For CPC, the NCBI eukaryotes' protein database was used and the e-value was set to ' $1 \mathrm{e}-10$ '. For Pfam-scan, each transcript was translated in all three possible frames and Pfam Scan (v1.3) was used to identify the occurrence of any of the known protein family domains documented in the Pfam database (release 27; used both Pfam A and Pfam B) (http:// pfam.xfam.org/). Any transcript with a Pfam hit was excluded from the following steps. Pfam searches used default parameters of -E 0.001 -domE 0.001 .

\section{Known miRNA alignment and novel miRNA prediction}

Mapped small RNA tags were used to look for known miRNA. MiRBase20.0 (http://www.mirbase.org/) [40] was used as a reference, modified software mirdeep2 [41] and srna-tools-cli were used to obtain the potential
miRNA and draw the secondary structures. Custom scripts were used to obtain the miRNA counts, as well as base bias at the first position of identified miRNA with certain length, and on each position of all identified miRNA respectively. To remove tags originating from protein-coding genes, repeat sequences, rRNA, tRNA, snRNA, and snoRNA, small RNA tags were mapped to RepeatMasker (http://www.repeatmasker.org/), Rfam 12.0 database (http://rfam.xfam.org/) [42] or those types of data (https://www.ncbi.nlm.nih.gov/) from goat.

The characteristic hairpin structure of miRNA precursors can be used to predict novel miRNA. The available software miREvo [43] and mirdeep2 [41] were integrated to predict novel miRNA through exploring the secondary structure, the Dicer cleavage site and the minimum free energy of small RNA tags unannotated in the former steps. At the same time, custom scripts were used to obtain the identified miRNA counts as well as base bias on the first position with certain length and on each position of all identified miRNA respectively.

\section{Quantification of gene expression level}

Cuffdiff (v2.1.1) [36] was used to calculate fragments per kb per million reads (FPKM) of both lncRNAs and coding genes in each sample. It was also used to provide statistical routines for determining differential expression in gene expression data using a model based on the negative binomial distribution. Transcripts or genes with a $P$-adjust $\leq 0.05$ [44] and fold change $\geq 2$ were described as differentially expressed between anagen and telogen. MiRNA expression levels were estimated by TPM (transcript per million) with the following criteria [45]: Normalization formula: Normalized expression $=$ actual miRNA count/total count of clean reads"1000000; Differential expression analysis of two groups was performed using the DESeq R package (1.8.3). The $P$-values were adjusted using the Benjamini and Hochberg method [44]. A corrected $P$-value of 0.05 was set as the threshold for significantly differential expression by default. Scatter plots were used to demonstrate differentially expressed miRNA between the two follicular stages.

\section{LncRNA target gene prediction}

To explore the function of IncRNAs, we first predicted the target genes of lncRNAs in cis and trans. The cis role refers to lncRNAs' action on neighboring target genes. In the present study, the coding genes from $100 \mathrm{~kb}$ upstream and downstream of an IncRNA were searched. The trans role refers to the influence of lncRNAs on other genes at the expression level. Pearson's correlation coefficients were calculated between expression levels of lncRNAs and mRNAs with custom scripts (Pearson correlation $\geq 0.95$ or $\leq-0.95$ ). 
Validation of gene expression in RNA-seq by quantitative PCR analysis

The total RNAs from the goats used for RNA-seq were also used for quantitative PCR analysis. For lncRNAs and mRNAs, the first-strand cDNA was obtained using a PrimeScript ${ }^{\text {Tw }}$ RT reagent Kit with gDNA Eraser (TAKARA, China), and then were subjected to quantification of the mRNAs and lncRNAs with $\beta$-actin as an endogenous control using $\mathrm{SYBR}^{\circ}$ Premix Ex Taq ${ }^{\mathrm{Tm}}$ II (TAKARA, China) on the Bio-Rad CFX96 Touch ${ }^{\text {Tw }}$ Real Time PCR Detection System. The quantitative PCR was performed using the following conditions: $95^{\circ} \mathrm{C}$ for $60 \mathrm{~s}$, 40 cycles of $95{ }^{\circ} \mathrm{C}$ for $10 \mathrm{~s}$, and the optimized annealing temperature for $30 \mathrm{~s}$. The primers and annealing temperatures for genes are listed in Additional file 1.

For miRNA quantitative PCR, the first-strand cDNA was obtained using Mir- $\mathrm{X}^{\mathrm{mi}}$ miRNA First Strand Synthesis Kit (Clontech, China), and then were subjected to quantification of miRNAs with U6 as an endogenous control as described above. The forward primer of specific miRNA was the sequence of the mature miRNA itself, the reverse was universal primer, which was provided in the kit. The primers for U6 were also provided in the kit. The quantitative PCR was performed on an CFX96 Touch $^{\text {tw }}$ (Bio-Rad, USA.) using the following conditions: $95{ }^{\circ} \mathrm{C}$ for $10 \mathrm{~s}, 40$ cycles of $95{ }^{\circ} \mathrm{C}$ for $5 \mathrm{~s}$, and the optimized annealing temperature for $20 \mathrm{~s}$.

Each stage (anagen and telogen) included at least 3 samples, and all reactions were performed in triplicate for each sample. Gene expression was quantified relative to endogenous gene expression using the comparative cycle threshold $(\triangle \mathrm{CT})$ method [46] through Bio-Rad CFX Manager 3.1 and Microsoft excel 2013. Differences in gene expression between the anagen and telogen were detected by independent sample $t$-test.

\section{Bioinformatics analysis}

Predicted interactions between miRNA and lncRNA were determined using miRanda. Predicted targets of differentially expressed miRNAs were determined using miRanda and targetscan (http://www.targetscan.org/ and http://www.microrna.org/microrna/). In addition, Pathway analysis was used to identify significant pathways for the differentially expressed genes according to the Kyoto Encyclopedia of Genes and Genomes (KEGG) (http://www.genome.jp/kegg/) [47]. We used KOBAS software (main parameter: blastx 1e-10; padjust: $\mathrm{BH}$ ) [48] to test the statistical enrichment of differentially expressed genes or lncRNA target genes in KEGG pathways.

\section{CeRNA network construction}

We predicted lncRNAs that might act as ceRNAs according to the following steps. (i) lncRNA screening:
IncRNAs that were up- or down-regulated fold change $>2.0$ and $P$-adjust value $<0.05$ were first retained; (ii) miRNAs associated with HF development and cycling were retained, including miR-148b, miR183, miR-184, miR-196a, miR-199a, miR-21, miR-24, miR-200, miR-200b, miR-203, miR-205, miR-214-3p, miR-221, miR-222, miR-31, miR-34a and miR-34c; (iii) lncRNA-mRNA interaction were obtained based on expression correlation coefficient (Pearson correlation $\geq 0.95$ ), the DEGs were retained; (iv) IncRNA-miRNA interactions were predicted by miRanda; (v) mRNAs targeted by miRNAs were predicted by miRanda and targetscan. The software Cytoscape 3.4 .0 was used to graphically visualize networks [49].

\section{Results}

Identification of IncRNAs in cashmere goat skin

In order to develop a comprehensive catalogue of lncRNAs in goat, a prerequisite was to integrate the high-quality and high-depth RNA-seq data set. To determine the orientation of transcripts accurately, we generated six transcriptomes covering different cycling stages of goat cashmere using the stranded sequencing method. A total of $644,884,566$ raw reads were produced from the Illumina PE 150 platform. After trimming to remove adaptor sequences, and discarding lowquality sequences, we retained $623,168,524$ clean reads (93.5 Gb, accounting for $96.63 \%$ of raw reads). Subsequently, we mapped the clean reads to the latest goat reference genome v2.0 (https://www.ncbi.nlm.nih.gov/ assembly/GCA_000317765.2) using the TOPHATCUFFLINKS pipeline [36]. Some filtering steps were conducted to retain bona fide lncRNAs (Fig. 1a). After coding potential analysis using the software $\mathrm{CNCI}, \mathrm{CPC}$ and Pfam-scan, 1108 lncRNA loci including 1010 long intergenic non-coding RNA (lincRNAs) and 98 anti-sense lncRNAs were identified (Fig. 1b) (Additional file 2). The sequence information of lncRNAs is shown in Additional file 3.

\section{Comparison of features of mRNAs and IncRNAs}

To comprehensively examine the differences between lncRNAs and the protein coding transcripts, comparative analysis was performed on gene structure, expression, and sequence conservation. The results showed that goat lncRNAs were slightly shorter than mRNAs in length distribution (Fig. 1c). Moreover, the number of exons and expression level were also less than that of mRNAs (Fig. 1d \& e).

\section{Identification of miRNAs in cashmere goat skin}

In order to identify miRNAs involved in the cashmere fiber cycle, four small RNA (sRNA) libraries representing anagen and telogen were constructed. Each 

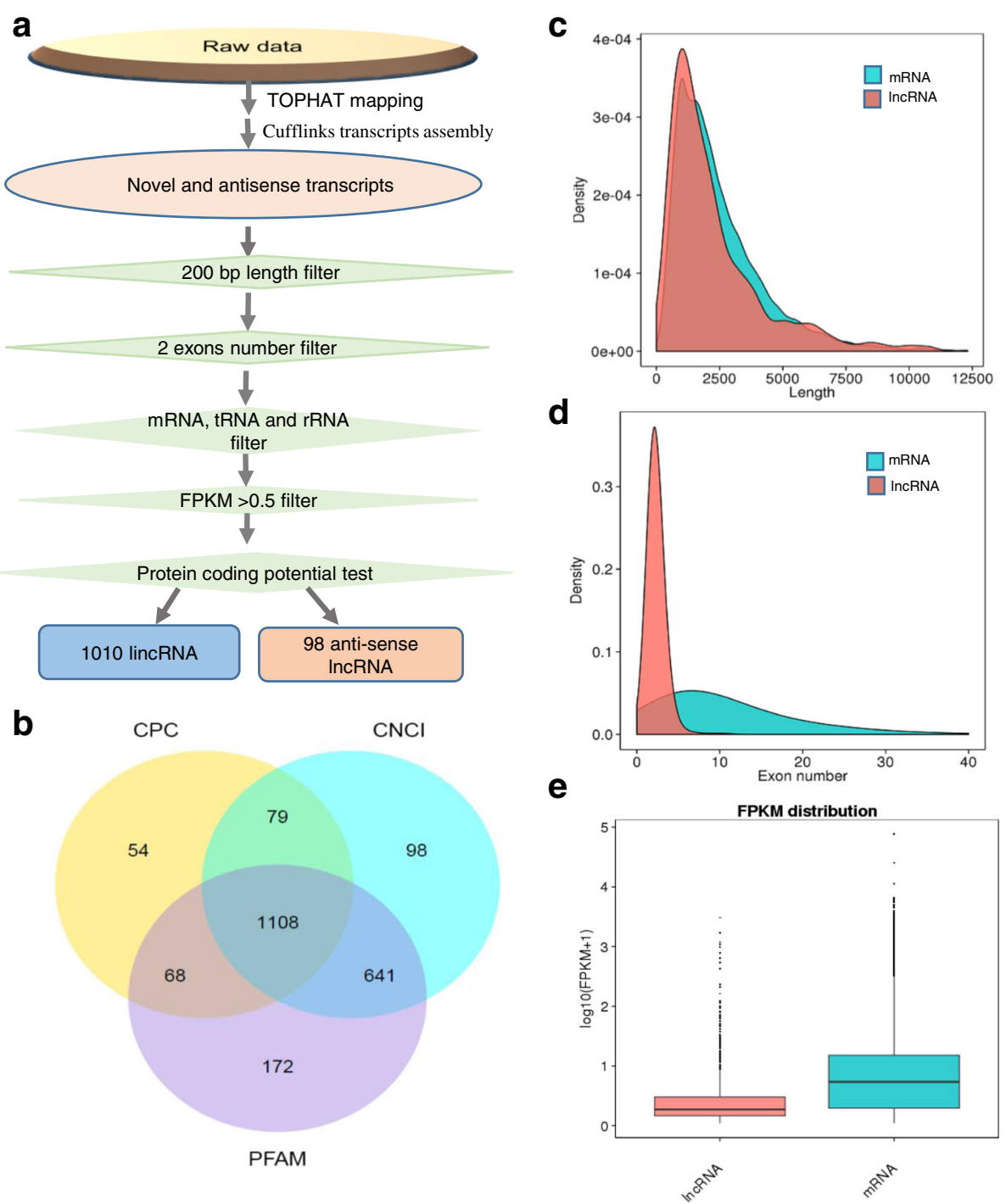

Fig. 1 Identification and characterization of long noncoding RNAs (IncRNAs) in Capra hircus. a The pipeline of IncRNA identification in Capra hircus. b Screening of the candidate IncRNAs in skin transcriptome. Venn diagrams of coding potential analysis by using stringent criteria. Three tools (CPC, CNCl and PFAM) were employed to analyze the coding potential of IncRNAs. Those simultaneously shared by three analytical tools were designated as candidate IncRNAs and used in subsequent analysis. c Distribution of transcript lengths in the IncRNAs and protein-coding transcripts. d Distribution of exon number in the IncRNAs and protein-coding transcripts. e Expression level indicated by $\log _{10}($ FPKM +1$)$ in the mRNAs and IncRNAs

library was a mixed pool from three adult cashmere goat skin samples. A total of 54,892,902 raw reads were obtained. After discarding the sequences shorter than $18 \mathrm{nt}$, eliminating low-quality sequences and removing contaminants formed by adapter-adapter ligation, reads without 3 ' ligation and insert tags were obtained. Collectively, a total of $54,013,578$ clean reads were retained for further analysis. Among these sequences, most were distributed in the 18$24 \mathrm{nt}$ range. The highest percentage of these sRNAs were 22 nt long, which is consistent with the common size of miRNAs. All the clean reads $(54,013,578)$ were aligned with the goat genome sequence using bowite v2.0.6 software [34]. Among them, 42,018,732 (77.79\%) clean reads were mapped in the goat genome. As a result, 411 annotated mature miRNAs from 259 precursors were identified (Additional file 4), which represented $10.86 \%$ of the total reads and $0.22 \%$ of the unique clean reads. Additionally, 130 novel mature miRNAs and 139 miRNA precursors were found using miREvo [43] and mirdeep2 (Additional file 5). 
Differentially expressed genes (DEGs) and non-coding RNAs (ncRNAs, miRNA and IncRNA)

The mRNA and lncRNA expression levels were analyzed using Cuffdiff v2.1.1 [36]. The overall expression levels of both lincRNAs and antisense-lncRNA were lower than that of protein-coding transcripts, which was consistent with a previous study [19]. Using edgeR (the threshold is usually set as Fold Change $\geq 2$ and $P$-adjust value $\leq 0.05$ ), the differentially expressed lncRNAs and genes between anagen and telogen were screened, resulting in 2492 DEGs (Additional file 6) and 198 differentially expressed lncRNAs (Additional file 7). Among these genes and lncRNAs, 1388 genes and 41 IncRNAs were upregulated, and 1104 genes and 157 IncRNAs were downregulated in anagen compared with telogen (Fig. 2).

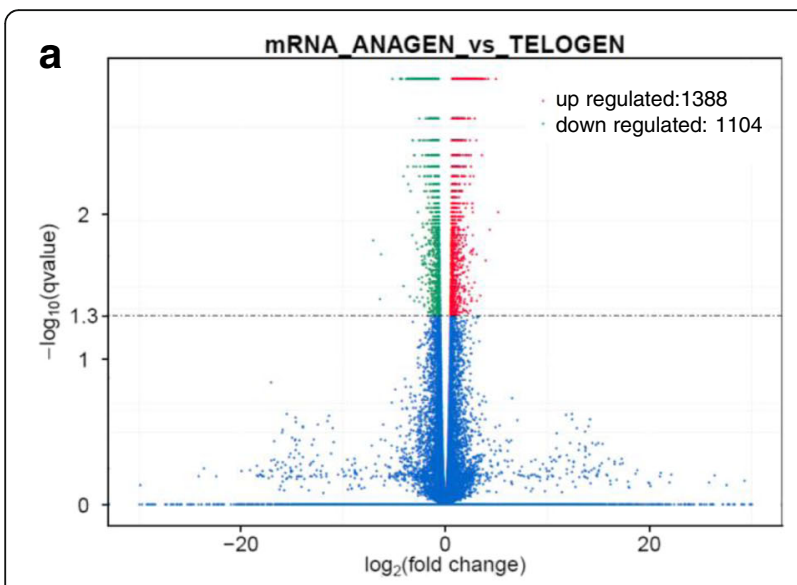

b

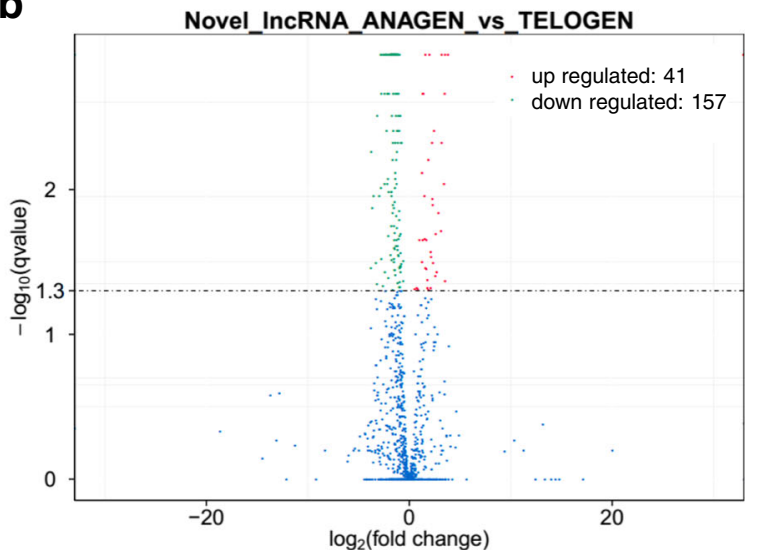

Fig. 2 Differentially expressed coding genes and IncRNAs in goat skin between anagen and telogen of HF cycle (Fold Change $\geq 2$ and $P$-adjust value $\leq 0.05$ ). a Differentially expressed coding genes. $\mathbf{b}$ Differentially expressed IncRNAs. Among these genes and IncRNAs, 1385 coding genes and 37 IncRNAs were upregulated, and 1104 coding genes and 156 IncRNAs were downregulated in anagen compared with telogen. Green dot indicates coding gene or IncRNA down regulated, red dot indicates coding gene or IncRNA up regulated in anagen compared with telogen
It was noteworthy that our analysis identified a set of genes belonging to keratin family member encoding genes (KRT) and keratin-associated protein (KAP), which were markedly up-regulated in anagen compared with telogen (Additional file 8). Previous studies have shown that KRT and KAP are major structural proteins of hair fiber and sheath, and their contents were important for fleece quality [50].

Meanwhile, a few lncRNAs were specifically expressed at a single developmental stage of cashmere cycling, such as Lnc_00092, Lnc_000183, Lnc_000406 and Lnc_000559, which showed telogen-specific expression, while Lnc_000173 showed anagen-specific expression, indicating that these lncRNAs could regulate cashmere cycling through their spatio-temporal expression.

As to miRNAs analysis, 21 known miRNAs and 2 novel miRNAs were found to have significantly different expression between anagen and telogen. Among them, 8 miRNAs were down-regulated and 15 miRNAs were upregulated compared with telogen (Additional file 9). Among these miRNAs, miR-214-3p, miR-196a and miR34c-5p had been reported with HF development and cycling functions [20, 24, 32].

To confirm the expression patterns of the genes, we randomly selected 5 lncRNAs, 6 mRNAs and 5 miRNAs and validated their expression patterns using quantitative realtime PCR (qRT-PCR). The results were in concordance with the RNA-seq data, suggesting that the expression patterns based on RNA-seq data were reliable (Fig. 3).

\section{KEGG analysis of DEGs}

KEGG analysis predicted that the DEGs were enriched in 274 pathways. The top 20 KEGG pathways with the highest representation of DEGs are shown in Fig. 4. Among the identified KEGG pathways, some belonged to conventional pathways associated with HF cycling, such as the WNT signaling pathway, ECM-receptor interaction, TGF- $\beta$ signaling pathway and VEGF signaling pathway. However, the oxidative phosphorylation pathway, proteasome pathway, metabolic pathway and lysine degradation pathways were up regulated, therefore may play an important role in HF cycling.

\section{The cis and trans role of IncRNAs in target genes}

To investigate the function of lncRNAs, the potential targets of lncRNAs in cis and trans were predicted. For the cis action of IncRNAs, we searched for proteincoding genes $100 \mathrm{~kb}$ upstream and downstream of the lncRNAs (Additional file 10). Interestingly, we detected HF cycling related genes such as WNT3A, HOXC13 and MSX2 which were located near the LNC_000972, LNC_000503 and LNC_000881 loci respectively (Table 1), suggesting that HF cycling may be regulated by the action of lncRNAs on neighboring protein-coding 

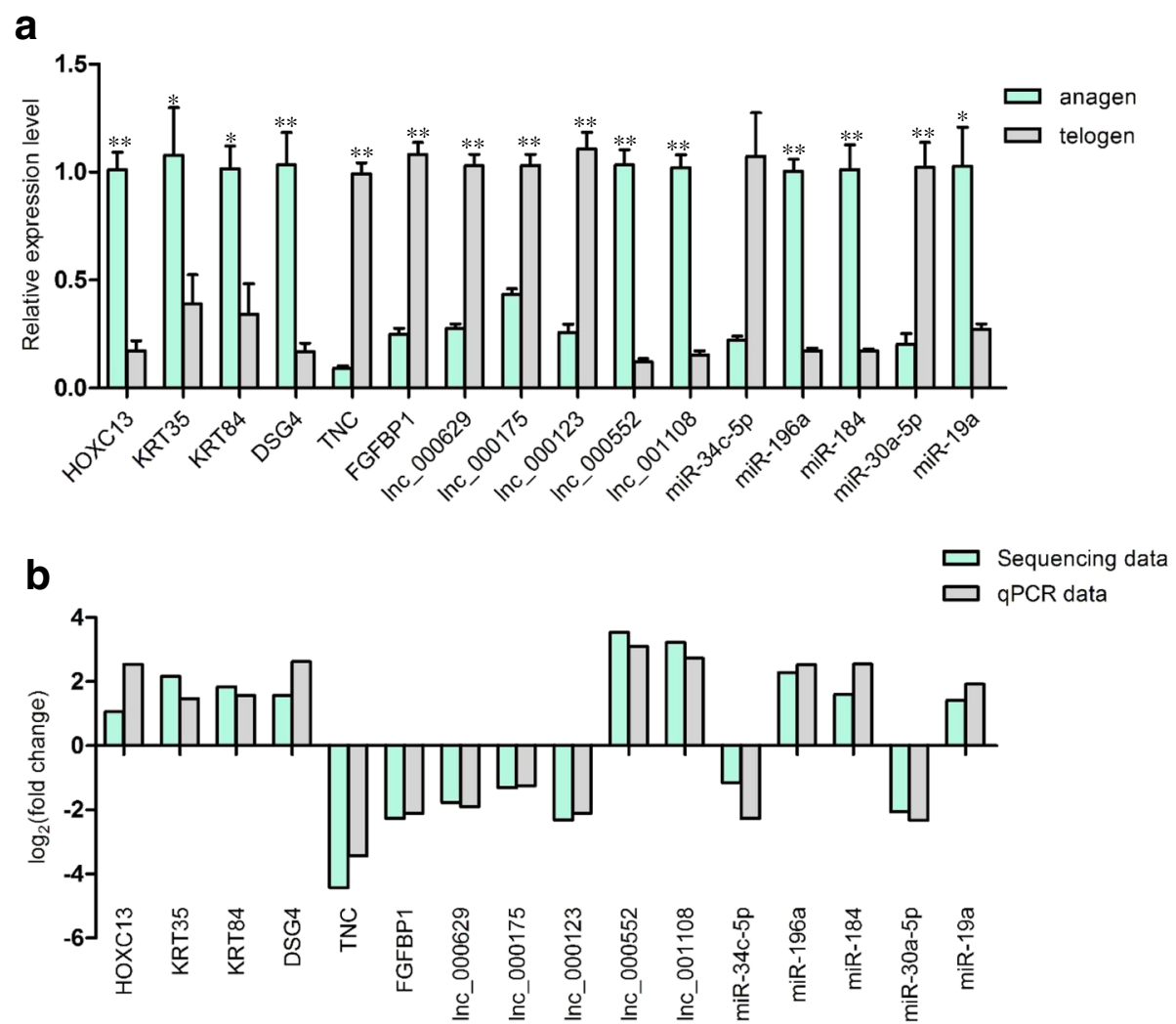

Fig. 3 Sequencing data validated by qPCR. a The expression level of differently expressed genes, miRNAs and IncRNAs validated by qPCR. Coding gene and IncRNA expression was quantified relative to the expression level of $\beta$-actin, miRNA expression was quantified relative to the expression level of U6 using the comparative cycle threshold $(\Delta \mathrm{CT})$ method. The data are expressed as the mean $\pm 1 \mathrm{SE}(n=3)$. ${ }^{*} P<0.05$, ** $P<0.01(\mathbf{b})$ Comparison the expression pattern of the sequencing data and GPCR data. $\log _{2}$ (fold change) $>0$ indicates the transcript up regulated in anagen compared to telogen. $\log _{2}$ (fold change) $<0$ indicates the transcript down regulated in anagen compared to telogen

genes. On the other hand, the trans role of 1108 lncRNAs in protein-coding genes was examined based on its expression correlation coefficient (Pearson correlation $\geq 0.95$ or $\leq-0.95$ ) (Additional file 11). As a result, we found that the genes related with HF biology might be targeted by a few lncRNAs (Table 1). Moreover, some lncRNAs, like lnc_000123, lnc_000188 and lnc_000203, target multiple KRTs and KAPs, which suggested their potential function on keratin regulation. Specifically, Lnc_000123 targeted APC, CTNNB1, NFATC1 and FZD1 (Fig. 5a), and lnc_001048 targeted BMPR1A, SMAD1, SMAD6, SMAD7 and TMEFF1 (Fig. 5b), which indicated that they might participate in the WNT or $\mathrm{BMP} / \mathrm{TGF} \beta$ pathways, respectively.

To further ascertain lncRNA-protein coding gene pairs belonging to both co-localization (cis action) and expression correlation (trans action) relationships, detailed examination was conducted. Eighty-four lncRNAprotein coding gene pairs that fulfilled these criteria were identified (Additional file 12). This finding suggested that lncRNAs act on their neighboring proteincoding genes to regulate gene expression.

\section{IncRNA as the precursor of miRNA and competing endogenous RNA (ceRNA)}

LncRNAs can be small RNA precursors and can also negatively regulate miRNA maturation [51]. When the lncRNA and miRNA sequencing data was combined, all the lncRNAs overlapped precursors of miRNAs from genomewide miRNA predictions. We found 12 lncRNAs that were possible precursors of 11 miRNAs (Additional file 13).

It has been shown that IncRNAs function as ceRNAs by binding to and sequestering specific miRNAs in both plants and animals [27], and some miRNAs have been reported to regulate HF development and cycling. We predicted lncRNA that might act as ceRNAs using strict rules (see Methods). It showed that some lncRNAs could bind to specific miRNAs that related to HF cycling to protect the target mRNAs from repression and thus play an important role in HF biology as ceRNAs, such as miR-34a-lnc_000181-GATA3 and miR-214-3plnc_000344-SMAD3 ceRNA networks (Fig. 6). Lnc_00 0181 and lnc_000344 serve as ceRNAs to upregulate GATA3 and SMAD3, respectively. The entire results were shown in Additional file 14. 


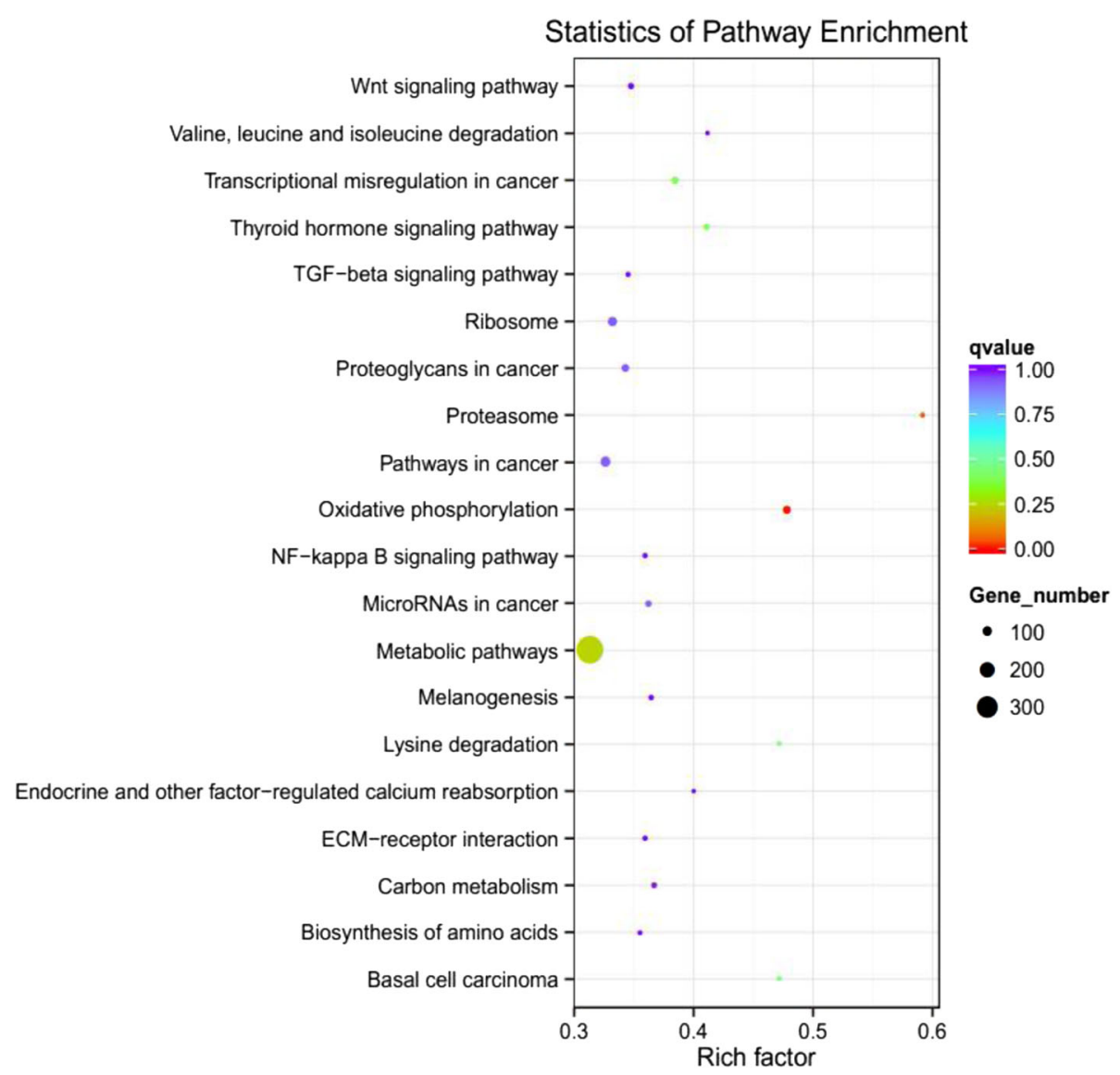

Fig. 4 The top 20 KEGG pathways of differentially expressed genes in goat skin between anagen and telogen. Rich factor indicates the ratio of DEGS enriched in the pathway among genes annotated in the pathway

\section{Discussion}

Hair is a characteristic feature of mammals and performs a variety of roles, such as thermal insulation, physical protection, camouflage, social interaction and sensory perception. HF consists of multiple different cell populations of neural crest, ectodermal or mesodermal origin, which are distinct in their location, function and gene and protein expression characteristics [52, 53]. Additionally, the HF is a stem cell-rich and uniquely dynamic mini-organ that under-goes continuous cycling throughout adult life, so it could serve as a perfect model for systems biology research and organ regeneration [54].

\section{Regulation of HF cycling}

The HF cycle has been traditionally subdivided into three stages: anagen (growth), catagen (cessation of growth) and telogen (resting), during which a number of morphological changes take place [55]. This cycle is regulated by complex and intricate interactions between the epithelial cells of the follicle and mesenchymal cells of the dermal papilla (DP) [5] and requires the spatiotemporal integration of multiple stimulatory and inhibitory signals [7, 56, 57], of which WNT and BMP/TGF $\beta$ are critical pathways [8, 58-60]. Corresponding with that, these pathways were also found in our current study. Note that previous studies mainly focused on human or mouse, however, cashmere growth is a seasonal phenomenon influenced by photoperiod, which is different to human or mouse. Studies have shown that melatonin plays a critical role in cashmere cycling and development under the control of photoperiod [61]. Melatonin may work through interacting with PRLR and DIO2 [62-64]. These two genes were found to have significantly different expression between anagen and telogen in this current study.

\section{Regulation of keratins during HF development}

The primary structural proteins of hair fibers are the hair keratins and the KAPs. During HF development, a notable feature is the orderly expression of the 
Table 1 LnCRNAs and its potential target genes that are involved in hair follicle cycling

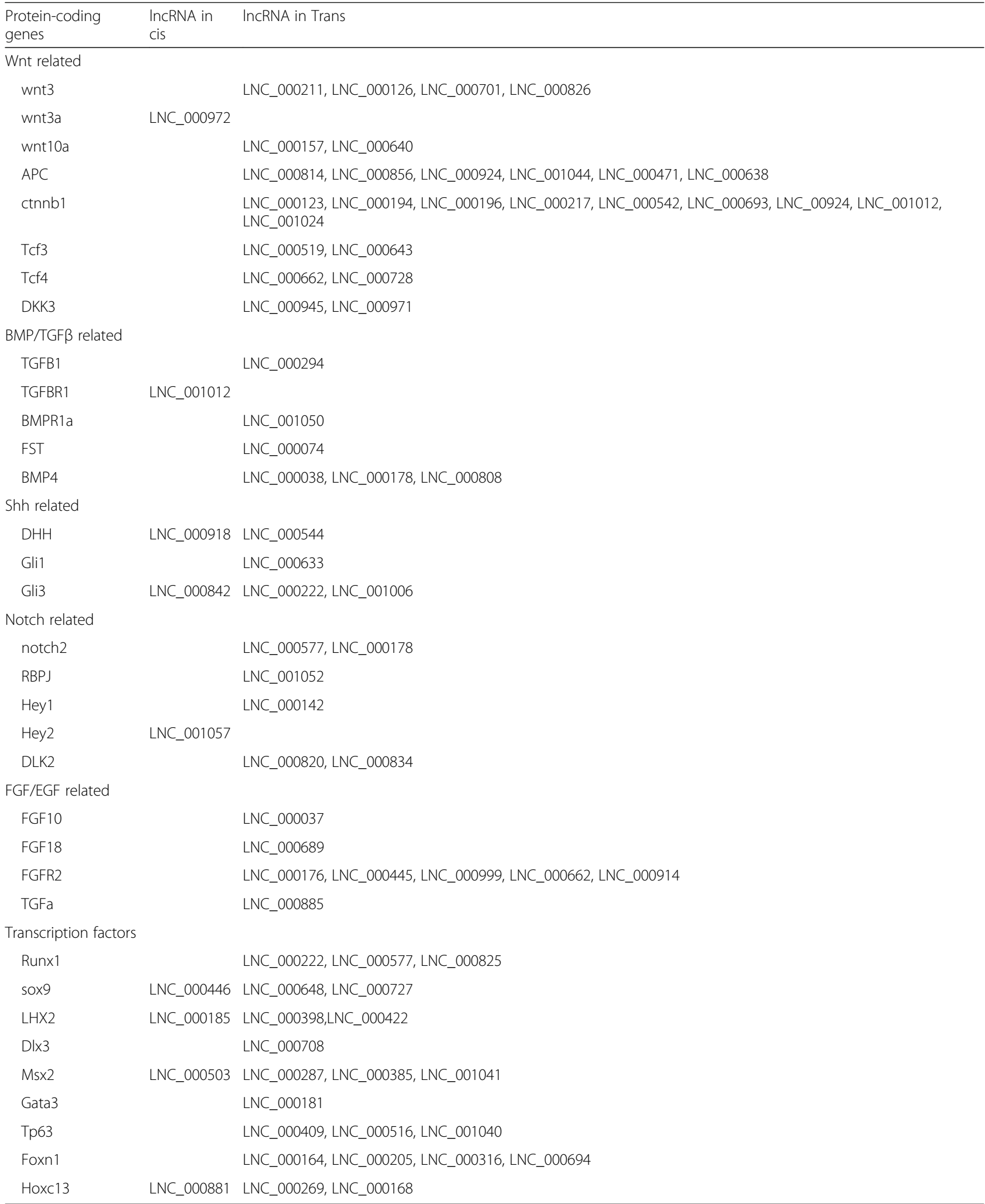



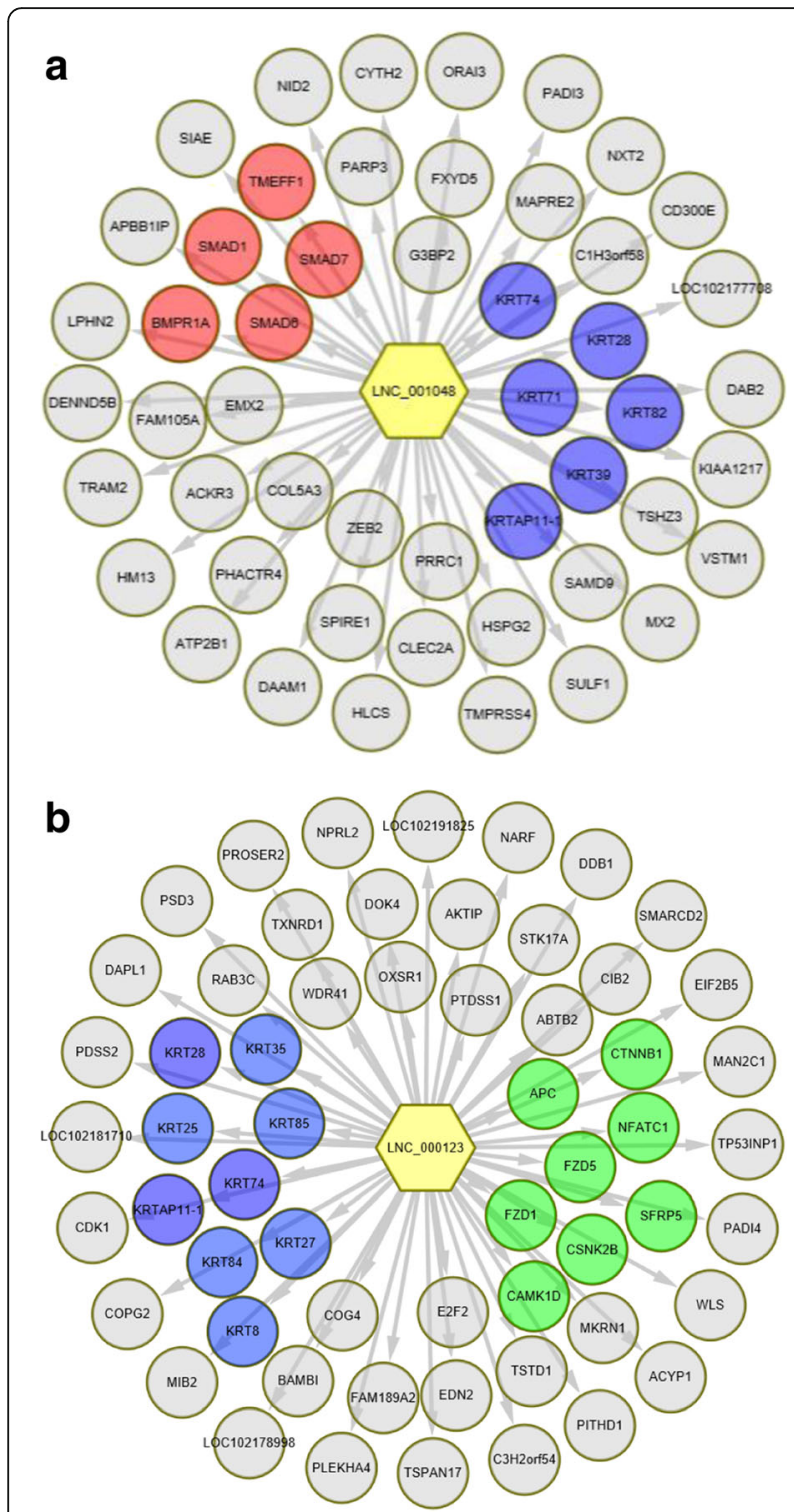

Fig. 5 The potential targets of Inc_001048 and Inc_000123. a The potential targets of Inc_001048. b The potential targets of Inc_00123. One IncRNA may target multiple targets, red color indicates BMP/TGF $\beta$ signals, green color indicates WNT signal and blue color indicate KRTs. Grey circles represent other potential targets beyond BMP and WNT signals or KRTs. DAVID Bioinformatics Resources 6.7 were used to generate the pathways. Arrows indicate effect direction

keratins and KAPs [53]. Corresponding with that, we found a set of genes belonging to keratin family members and KAPs, which were markedly upregulated in anagen vs. telogen. Keratins are intermediate filament proteins that have essential functions in maintaining the structural integrity of the epidermis and its appendages. Cell-specific keratin expression and organization impact on cell growth, migration and invasion. Their expression is under strict control to produce keratins that are optimally adapted to their environment [50, 65]. Transcription factors and other regulatory genes like $L E F 1, S P 1$, HOXC13, FOXN1, DSG4, AP1 and AP2 are critical factors that could regulate keratins [66-69]. Consistent with this, significant differences were observed between the expression of these genes in anagen and telogen, which verified their interactions in HF.

MiRNAs and IncRNAs play an important role in HF cycling Beyond coding genes, ncRNAs play an indispensable role in HF biology. Previous studies have identified a few miRNAs and revealed their function in HF morphogenesis and development, such as miR-214, miR21, miR-24 and miR-196a [22-24]. However, these studies only focused on the HF biology of humans and mice, little was known about cashmere goat HF biology, specifically cashmere cycling. We found that miR-214 and miR-196a might also play an important role in HF cycling of cashmere goat. Other studies revealed that lncRNAs play important regulatory roles in gene expression and contribute to skin biology [25], whereas there had been no related reports on cashmere cycling. In this study, we have identified lncRNAs in different HF stages of cashmere. We identified 1108 lncRNAs in total, some of which were adjacent or co-expressed with HF development related genes, which indicated their possible functions on $\mathrm{HF}$ cycling. These will greatly enrich the lncRNA database in Cashmere goat.

\section{LncRNA could function as ceRNA during HF cycling}

Nowadays, the influence of lncRNAs upon miRNA function is also rapidly emerging. In some cases, lncRNA stability is reduced through the interaction of specific miRNAs. In other cases, lncRNAs can act as miRNA decoys, with the sequestration of miRNA favoring expression of repressed target mRNAs. Other lncRNAs depress gene expression by competing with miRNAs for interaction with shared target mRNAs. Finally, some lncRNAs can produce miRNAs, leading to repression of target mRNAs [27, 28, 70]. These miRNA-lncRNA interactions are central to mammalian physiological and pathological processes [71, 72].

In current study, we constructed the ceRNA networks jointed by lncRNAs, miRNAs and mRNAs based on lncRNA and miRNA sequencing data. To enhance data reliability, defined rules were set to screen candidate ceRNAs. Our analysis has suggested that lncRNAs harbor potential miRNA recognition elements and participate in a complex ceRNA network. The network brings to light an unknown miRNA regulatory network in cashmere cycling. It also 

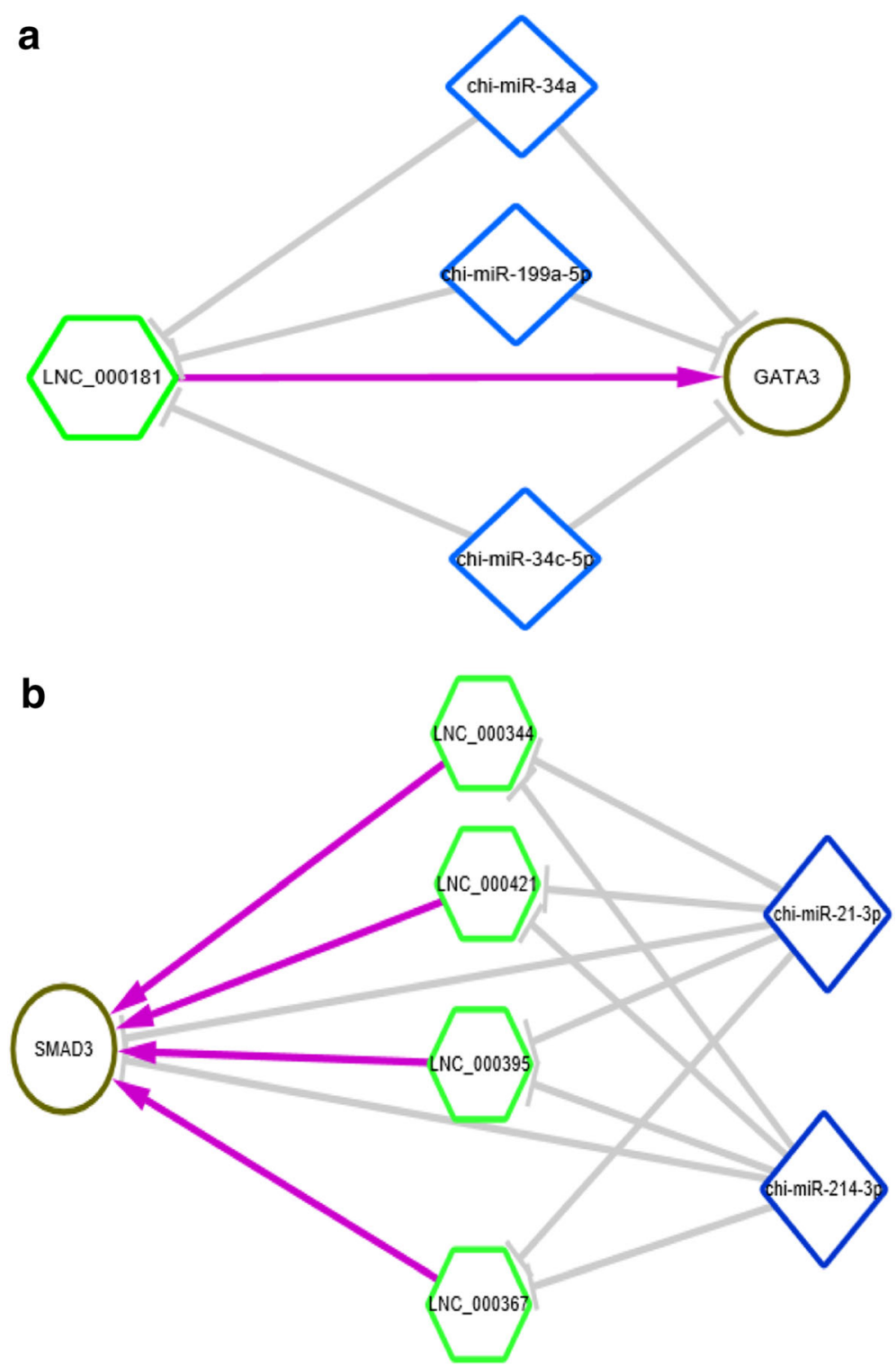

Fig. 6 LncRNA function as ceRNA. a Inc_000181 serve as ceRNA to upregulate GATA3, (b) Inc_000344, Inc_000367, Inc_000395 and Inc_000421 may serve as ceRNAs to upregulate SMAD3. Hexagon indicates differently expressed IncRNA between anagen and telogen. Rhombus indicates miRNA involved in HF cycling and development. Circle indicates differently expressed gene between anagen and telogen. LncRNA-mRNA interactions were obtained based on its expression correlation coefficient (Pearson correlation $\geq 0.95$ ). LncRNA-miRNA interactions were predicted by miRanda, mRNAs targeted by miRNAs were predicted by miRanda and targetscan. Gray line indicates negative regulation, purple line indicates positive regulation

suggests that lncRNAs may play crucial roles in cashmere cycling and maintenance.

\section{Conclusions}

In conclusion, in this study, we present the first data on the IncRNA of cashmere cycling. Combined with the miRNA sequence data, the ceRNA networks were constructed, which expands our knowledge about lncRNA and miRNA biology and contributes to the annotation of the goat genome as well.

\section{Additional files}

Additional file 1: Primers used in quantitative PCR analysis. (XLSX $9 \mathrm{~kb}$ )

Additional file 2: Novel IncRNA information. (XLSX $85 \mathrm{~kb}$ )

Additional file 3: The sequence of IncRNA. (TXT $2937 \mathrm{~kb}$ )

Additional file 4: Annotated mature miRNAs and precursors. (XLSX $17 \mathrm{~kb}$ )

Additional file 5: Novel mature miRNA sequence and precursor sequence. (TXT $14 \mathrm{~kb}$ )

Additional file 6: Differentially expressed genes between anagen and telogen. (XLSX $229 \mathrm{~kb}$ ) 
Additional file 7: Differentially expressed IncRNA between anagen and telogen. (XLSX $26 \mathrm{~kb}$ )

Additional file 8: Differentially expressed KRTS and KAPs. (XLSX $14 \mathrm{~kb}$ ) Additional file 9: Differentially expressed miRNAs between anagen and telogen. (XLSX $15 \mathrm{~kb}$ )

Additional file 10: The protein-coding genes within $100 \mathrm{k}$ upstream and downstream of an IncRNA. (XLSX $310 \mathrm{~kb}$ )

Additional file 11: Pearson correlations between protein-coding genes and IncRNAs. (XLSX $1804 \mathrm{~kb})$

Additional file 12: LncRNA-protein coding gene pairs with both colocalization and correlation relationships. (XLSX $12 \mathrm{~kb}$ )

Additional file 13: LncRNA as miRNA precursor. (XLSX $10 \mathrm{~kb}$ )

Additional file 14: Potential IncRNAs function as competing endogenous RNAs (ceRNAs) duing hair follicle cycling. (XLSX 17 kb)

\section{Abbreviations}

DEG: Differentially expressed gene; HF: Hair follicle; KAP: Keratin-associated protein; KEGG: Kyoto encyclopedia of genes and genomes; KRT: Keratin family member encoding gene; lincRNA: Long intergenic non-coding RNA; IncRNA: Long non-coding RNA; miRNA: microRNA; ncRNA: Non-coding RNA; PAGE: Polyacrylamide gel electrophoresis; qRT-PCR: Quantitative real-time PCR

\section{Acknowledgements}

Not applicable.

\section{Funding}

This research was supported by the National Natural Science Foundation of China (No. 31472068 \& No. 31201769).

\section{Availability of data and materials}

The data supporting the findings of this study are available within its supplementary information.

\section{Authors' contributions}

WSH conceived the idea of this research, performed data analysis and drafted the manuscript. GW participated in the data analysis and graphics. LZX conducted qPCR validation. GY and JBL conducted animal experiments and surgical processes. QL provided the experimental animals. ZZY and WX provided the experimental environment and coordination. All authors have read and approved the final manuscript.

\section{Ethics approval}

All the experimental procedures with goats used in the present study had been given prior approval by the Experimental Animal Manage Committee of Northwest A\&F University (2011-31101684).

\section{Consent for publication}

Not applicable.

\section{Competing interests}

The authors declare that they have no competing interests.

\section{Publisher's Note}

Springer Nature remains neutral with regard to jurisdictional claims in published maps and institutional affiliations.

\section{Author details}

'College of Animal Science \& Technology, Northwest A\&F University, Yangling, Shaanxi 712100, China. ${ }^{2}$ Life Science Research Center, Yulin University, Yulin, Shaanxi 719000, China.
Received: 21 December 2016 Accepted: 2 October 2017

Published online: 11 October 2017

\section{Reference}

1. Berger J, Buuveibaatar B, Mishra C. Globalization of the cashmere market and the decline of large mammals in central Asia. Conserv Biol. 2013;27(4): 679-89.

2. Mcdonald BJ, Hoey WA, Hopkins PS. Cyclical fleece growth in cashmere goats. Aus J Agric Res. 1987;38(3):597-609.

3. Norton BW, Klören WRL. Measurement of the components of the cashmere growth cycle in Australian cashmere goats. Small Ruminan Res. 1995;17(3): 263-8.

4. Kloren W, Norton B, Waters M. Fleece growth in Australian cashmere goats. III. The seasonal patterns of cashmere and hair growth, and association with growth hormone, prolactin and thyroxine in blood. Crop Pasture Sci. 1993; 44(5):1035-50.

5. Sennett R, Rendl M. Mesenchymal-epithelial interactions during hair follicle morphogenesis and cycling. Semin Cell Dev Biol. 2012;23(8):917-27.

6. Yang CC, Cotsarelis G. Review of hair follicle dermal cells. J Dermatol Sci. 2010:57(1):2-11.

7. Lee J, Tumbar T. Hairy tale of signaling in hair follicle development and cycling. Semin Cell Dev Biol. 2012;23(8):906-16.

8. Tsai SY, Sennett R, Rezza A, Clavel C, Grisanti L, Zemla R, Najam S, Rendl M. Wnt/beta-catenin signaling in dermal condensates is required for hair follicle formation. Dev Biol. 2014;385(2):179-88.

9. Qiu W, Lei M, Tang H, Yan H, Wen X, Zhang W, Tan R, Wang D, Wu J. Hoxc13 is a crucial regulator of murine hair cycle. Cell Tissue Res. 2016; 364(1):149-58.

10. Mukhopadhyay A, Krishnaswami SR, Cowing-Zitron C, Hung NJ, ReillyRhoten H, Burns J, Yu BD. Negative regulation of Shh levels by Kras and Fgfr2 during hair follicle development. Dev Biol. 2013;373(2):373-82.

11. Rishikaysh P, Dev K, Diaz D, Qureshi WM, Filip S, Mokry J. Signaling involved in hair follicle morphogenesis and development. Int J Mol Sci. 2014;15(1):1647-70

12. Leirós GJ, Attorresi Al, Balañá ME. Hair follicle stem cell differentiation is inhibited through cross-talk between Wnt/ß-catenin and androgen signalling in dermal papilla cells from patients with androgenetic alopecia. Br J Dermatol. 2012;166(5):1035-42.

13. Samuelov L, Sprecher E, Tsuruta D, Bíró T, Kloepper JE, Paus R, P-cadherin regulates human hair growth and cycling via canonical Wnt signaling and transforming growth factor- $\beta 2$. J Invest Dermatol. 2012;132(10):2332-41.

14. Jahoda CA, Christiano AM. Niche crosstalk: intercellular signals at the hair follicle. Cell. 2011;146(5):678-81.

15. Mashanov VS, Zueva OR, García-Arrarás JE. Transcriptomic changes during regeneration of the central nervous system in an echinoderm. BMC Genomics. 2014:15(1):357.

16. Kenneth $F$, Jérémie $B$, Pierre-Luc $M$, Mathieu $L$, Jean-Charles $G$, Anie $P$, Stéphane R. BMP-2 functions independently of SHH signaling and triggers cell condensation and apoptosis in regenerating axolotl limbs. BMC Dev Biol. 2010;10:15

17. Wang KC, Chang HY. Molecular mechanisms of long noncoding RNAs. Mol Cell. 2011:43(6):904-14.

18. Huang Y, Shen XJ, Zou Q, Wang SP, Tang SM, Zhang GZ. Biological functions of microRNAs: a review. J Physiol Biochem. 2011;67(1):129-39.

19. Ren H, Wang G, Chen L, Jiang J, Liu L, Li N, Zhao J, Sun X, Zhou P. Genomewide analysis of long non-coding RNAs at early stage of skin pigmentation in goats (Capra Hircus). BMC Genomics. 2016;17:67

20. Andl T, Murchison EP, Liu F, Zhang Y, Yunta-Gonzalez M, Tobias JW, Andl CD, Seykora JT, Hannon GJ, Millar SE. The miRNA-processing enzyme dicer is essential for the morphogenesis and maintenance of hair follicles. Curr Biol. 2006:16(10):1041-9.

21. Teta M, Choi YS, Okegbe T, Wong G, Tam OH, Chong MM, Seykora JT, Nagy A, Littman DR, Andl T, et al. Inducible deletion of epidermal dicer and Drosha reveals multiple functions for miRNAs in postnatal skin. Development. 2012:139(8):1405-16

22. Amelio I, Lena AM, Bonanno E, Melino G, Candi E. miR-24 affects hair follicle morphogenesis targeting Tcf-3. Cell Death Dis. 2013:4:e922.

23. Ahmed MI, Mardaryev AN, Lewis CJ, Sharov AA, Botchkareva NV. MicroRNA21 is an important downstream component of BMP signalling in epidermal keratinocytes. J Cell Sci. 2011;124(Pt 20):3399-404. 
24. Ahmed MI, Alam M, Emelianov VU, Poterlowicz K, Patel A, Sharov AA, Mardaryev AN, Botchkareva NV. MicroRNA-214 controls skin and hair follicle development by modulating the activity of the Wnt pathway. J Cell Biol. 2014;207(4):549-67.

25. Wan DC, Wang KC. Long noncoding RNA: significance and potential in skin biology. Cold Spring Harb Perspect Med. 2014;4(5):a015404

26. Lin CM, Liu Y, Huang K, Chen XC, Cai BZ, Li HH, Yuan YP, Zhang H, Li Y. Long noncoding RNA expression in dermal papilla cells contributes to hairy gene regulation. Biochem Biophys Res Commun. 2014;453(3):508-14.

27. Guil S, Esteller M. RNA-RNA interactions in gene regulation: the coding and noncoding players. Trends Biochem Sci. 2015;40(5):248-56.

28. Salmena L, Poliseno L, Tay $Y$, Kats L, Pandolfi PP. A ceRNA hypothesis: the Rosetta stone of a hidden RNA language? Cell. 2011;146(3):353-8.

29. Terai G, Iwakiri J, Kameda T, Hamada M, Asai K. Comprehensive prediction of IncRNA-RNA interactions in human transcriptome. BMC Genomics. 2016 17(Suppl 1):12.

30. Guo L, Zhao Y, Yang S, Zhang H, Wu Q, Chen F. An integrated evolutionary analysis of miRNA-IncRNA in mammals. Mol Biol Rep. 2014;41(1):201-7.

31. Li N, Ponnusamy M, Li MP, Wang K, Li PF. The role of MicroRNA and LncRNA-MicroRNA interactions in regulating ischemic heart disease. J Cardiovasc Pharmacol Ther. 2016;22(2):105-11.

32. Yuan C, Wang X, Geng R, He X, Qu L, Chen Y. Discovery of cashmere goat (Capra Hircus) microRNAs in skin and hair follicles by Solexa sequencing. BMC Genomics. 2013;14(1):511.

33. Ewing B, Hillier LD, Wendl MC, Green P. Base-calling of automated sequencer traces using PHRED. I. Accuracy assessment. Genome Res. 1998; 8(3):175-85

34. Langmead RBB, Trapnell C, Pop M, Salzberg SL. Ultrafast and memoryefficient alignment of short DNA sequences to the human genome. Genome Biol. 2009;10(3):R25.

35. Trapnell C, Pachter L, Salzberg SL. TopHat: discovering splice junctions with RNA-Seq. Bioinformatics. 2009;25(9):1105-11.

36. Trapnell C, Roberts A, Goff L, Pertea G, Kim D, Kelley DR, Pimentel H, Salzberg SL, Rinn JL, Pachter L. Differential gene and transcript expression analysis of RNA-seq experiments with TopHat and Cufflinks. Nat Protoc. 2012;7(3):562-78.

37. Kong L, Zhang Y, Ye ZQ, Liu XQ, Zhao SQ, Wei L, Gao G. CPC: assess the protein-coding potential of transcripts using sequence features and support vector machine. Nucleic Acids Res. 2007;35:W345-9.

38. Punta M, Coggill PC, Eberhardt RY, Mistry J, Tate J, Boursnell C, Pang N, Forslund K, Ceric G, Clements J, et al. The Pfam protein families database. Nucleic Acids Res. 2012;40(D1):D290-301.

39. Sun L, Luo HT, Bu DC, Zhao GG, Yu KT, Zhang CH, Liu YN, Chen RS, Zhao Y. Utilizing sequence intrinsic composition to classify protein-coding and long non-coding transcripts. Nucleic Acids Res. 2013;41(17):e166.

40. Kozomara A, Griffithsjones S. miRBase: annotating high confidence microRNAs using deep sequencing data. Nucleic Acids Res. 2014; 42(Database issue):68-73.

41. Friedländer MR, Mackowiak SD, Li N, Chen W, Rajewsky N. miRDeep2 accurately identifies known and hundreds of novel microRNA genes in seven animal clades. Nucleic Acids Res. 2012;40(1):37-52

42. Nawrocki EP, Burge SW, Bateman A, Daub J, Eberhardt RY, Eddy SR, Floden EW, Gardner PP, Jones TA, Tate J, Finn RD. Rfam 12.0: updates to the RNA families database. Nucleic Acids Res. 2015;43(Database issue):D130-7.

43. Wen $M$, Shen $Y$, Shi S, Tang T. miREvo: an integrative microRNA evolutionary analysis platform for next-generation sequencing experiments. BMC Bioinformatics. 2012;13:140.

44. Storey JD. The positive false discovery rate: a Bayesian interpretation and the q-value. Ann Stat. 2003;31(6):2013-35.

45. Zhou L, Chen J, Li Z, Li X, Hu X, Huang Y, Zhao X, Liang C, Wang Y, Sun L, et al. Integrated profiling of MicroRNAs and mRNAs: MicroRNAs located on Xq27.3 associate with clear cell renal cell carcinoma. PLoS One. 2010;5(12): e15224.

46. Schmittgen TD, Livak KJ. Analyzing real-time PCR data by the comparative C(T) method. Nat Protoc. 2008;3(6):1101-8.

47. Kanehisa M, Araki M, Goto S, Hattori M, Hirakawa M, Itoh M, Katayama T, Kawashima S, Okuda S, Tokimatsu T. KEGG for linking genomes to life and the environment. Nucleic Acids Res. 2008;36(Database issue):D480-4.

48. Mao X, Cai T, Olyarchuk JG, Wei L. Automated genome annotation and pathway identification using the KEGG Orthology $(\mathrm{KO})$ as a controlled vocabulary. Bioinformatics. 2005;21(19):3787-93.
49. Shannon P, Markiel A, Ozier O, Baliga NS, Wang JT, Ramage D, Amin N, Schwikowski B, Ideker T. Cytoscape: a software environment for integrated models of biomolecular interaction networks. Genome Res. 2003;13(11): 2498-504.

50. Magin TM, Vijayaraj P, Leube RE. Structural and regulatory functions of keratins. Exp Cell Res. 2007;313(10):2021-32.

51. Shi Y, Wang Y, Luan W, Wang P, Tao T, Zhang J, Qian J, Liu N, You Y. Long non-coding RNA H19 promotes Glioma cell invasion by deriving miR-675. PLoS One. 2014;9(1):e86295.

52. Hsu YC, Pasolli HA, Fuchs E. Dynamics between stem cells, niche, and progeny in the hair follicle. Cell. 2011;144(1):92-105.

53. Rogers GE. Hair follicle differentiation and regulation. Int J Dev Biol. 2004; 48(2-3):163-70.

54. Al-Nuaimi Y, Baier G, Watson RE, Chuong CM, Paus R. The cycling hair follicle as an ideal systems biology research model. Exp Dermatol. 2010;19(8):707-13.

55. Paus R, Foitzik K. In search of the "hair cycle clock": a guided tour. Differentiation. 2004;72(9-10):489-511.

56. Al-Nuaimi Y, Goodfellow M, Paus R, Baier G. A prototypic mathematical model of the human hair cycle. J Theor Biol. 2012;310:143-59.

57. Baker RE, Murray PJ. Understanding hair follicle cycling: a systems approach. Curr Opin Genet Dev. 2012;22(6):607-12.

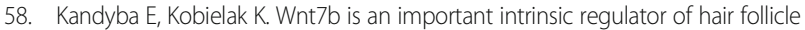
stem cell homeostasis and hair follicle cycling. Stem Cells. 2014;32(4):886-901.

59. Lin KK, Kumar V, Geyfman M, Chudova D, Ihler AT, Smyth P, Paus R, Takahashi JS, Andersen B. Circadian clock genes contribute to the regulation of hair follicle cycling. PLoS Genet. 2009:5(7):e1000573.

60. Guha U, Mecklenburg L, Cowin P, Kan L, O'Guin WM, D'Vizio D, Pestell RG, Paus $\mathrm{R}$, Kessler JA. Bone morphogenetic protein Signaling regulates postnatal hair follicle differentiation and cycling. Am J Pathol. 2004;165(3):729-40.

61. Klören W, Norton BW. Melatonin and fleece growth in Australian cashmere goats. Small Ruminant Res. 1995;17(2):179-185.

62. Emesih GC, Newton GR, Teh TH, Zia JH. Effects of photoperiod and continuous administration of melatonin on plasma concentrations of prolactin in cashmere goats. Small Ruminant Res. 1993;11(3):247-56.

63. Yue CW, Du LX, Wei Z, Zhu XP, Kong XH, Jia ZH. Expression of Prolactin receptor mRNA after melatonin manipulated in cashmere goats skin during cashmere growth. Asian-Australas J Anim Sci. 2010;23(10):1291-8.

64. Jianning HE, Huang DW, Ran DI, Wang JX, Chu MX, Liu QY, Wenping HU, Wang XY, Pan ZY. Polymorphism of exon 2 of DIO2 gene and its association withseasonal reproduction in sheep. Turk J Vet Anim Sci. 2016;40(2):142-9.

65. Loschke F, Seltmann K, Bouameur JE, Magin TM. Regulation of keratin network organization. Curr Opin Cell Biol. 2015;32:56-64.

66. Blumenberg M. Transcriptional regulation of keratin gene expression. In: Intermediate Filaments. Springer: Boston, MA; 2006. p. 93-109.

67. Powell BC, Nesci A, Rogers GE. Regulation of keratin gene expression in hair follicle differentiation. Ann N Y Acad Sci. 1991;642:1-20.

68. Jave-Suarez LF, Winter H, Langbein L, Rogers MA, Schweizer J. HOXC13 is involved in the regulation of human hair keratin gene expression. J Biol Chem. 2002;277(5):3718-26.

69. Bazzi H, Demehri S, Potter CS, Barber AG, Awgulewitsch A, Kopan R, Christiano AM. Desmoglein 4 is regulated by transcription factors implicated in hair shaft differentiation. Differentiation. 2009;78(5):292-300.

70. Rogler LE, Kosmyna B, Moskowitz D, Bebawee R, Rahimzadeh J, Kutchko K, Laederach A, Notarangelo LD, Giliani S, Bouhassira E, et al. Small RNAs derived from IncRNA RNase MRP have gene-silencing activity relevant to human cartilage-hair hypoplasia. Hum Mol Genet. 2014;23(2):368-82.

71. Wu Q, Guo L, Jiang F, Li L, Li Z, Chen F. Analysis of the miRNA\&ndash; mRNA\&ndash;IncRNA networks in ER+ and ER- breast cancer cell lines. J Cell Mol Med. 2015;19(12):2874-87.

72. Xia T, Liao Q, Jiang X, Shao Y, Xiao B, Xi Y, Guo J. Long noncoding RNA associated-competing endogenous RNAs in gastric cancer. Sci Rep. 2014;4:6088. 